\title{
Beyond the Special and General Relativity Theories The Micro-quanta Paradigm
}

\author{
Maurizio Michelini ${ }^{1}$ \\ ${ }^{1}$ ENEA - Casaccia Research Centre - via Anguillarese 301, Rome, Italy \\ Correspondence: Maurizio Michelini, ENEA - Casaccia Research Centre - via Anguillarese 301, Rome, Italy. \\ E-mail: m_michelini@alice.it
}

Received: April 2, $2012 \quad$ Accepted: April 18, $2012 \quad$ Online Published: May 1, 2012

doi:10.5539/apr.v4n2p210 URL: http://dx.doi.org/10.5539/apr.v4n2p210

\begin{abstract}
Despite the arbitrary postulate of empty space (which was as arbitrary as the old aether), Albert Einstein obtained through the conceptually powerful postulate of the constancy of light velocity in any reference system, the relativistic equations of motion. The kinematic basis of Special relativity was responsible of several physical paradoxes that accompanied the growing reliability of SR along the XX century. Conversely, in the Micro-quanta paradigm the particle dynamics is directly derived in dynamical conditions from Compton's collisions between particles and the isotropic flux $\phi_{o}$ of micro-waves filling the space with speed $c$ and energy $\mathrm{E}_{\mathrm{o}}$. When considering the proper Doppler function for waves incident forwards and behind any moving particle, the balance between the two opposite momenta gives the momentum predicted by SR and defines also the rest-mass $m_{i}$ of the particle, which results (equivalent) to the energy $m_{\mathrm{i}} \mathrm{c}^{2}=\mathrm{N}_{\mathrm{i}} \mathrm{E}_{\mathrm{o}}$, where $\mathrm{N}_{\mathrm{i}}=\left(\sigma_{\mathrm{i}} \tau_{\mathrm{o}} \phi_{\mathrm{o}}\right)$ is the number of quanta colliding simultaneously with the particle during the interaction time $\tau_{\mathrm{o}}$. In the M-Q paradigm the uniform flux fills everywhere the space, so particles "measure" their effective velocity through the momentum of Compton's collisions. The old inertial frames of reference become unnecessary. Any particle moving uniformly in strait-line is shown to collide with micro-quanta without receiving any net force (Principle of inertia). When the particle velocity is not constant and uniform during the interaction time, the local energy density $\ni_{0}=\phi_{0} \mathrm{E}_{0} / \mathrm{c}$ gives rise to the inertial forces. Since two particles immersed in the quantum flux always show mutual shielding, they feel a pushing force which is recognised to be the gravitational force. Thus, the concept of "gravitational mass" is discarded. This inevitably requires to re-examine the fundamentals of General relativity which appears inadequate to explain the recent observations of the accelerating universe achieved through the Ia supernovae measurements. Even the Strong nucleonic force and the Weak force on electrons at very short distances are shown to originate from mutual shielding in the M-Q paradigm. Despite micro-quanta are very small, they do not lose the fundamental characteristics of the quantum waves. The very little quantum energy $E_{o}=h_{o} v_{o}$ is coupled to a very little action constant and a very small quantum wavelength $\lambda_{\mathrm{o}}$ (of the order of the Planck's length), so that the number of quanta simultaneously incident on a nucleon is about $\mathrm{N}_{\mathrm{n}} \approx 10^{50}$. This explains why the inertial forces were for three centuries (from Newton up to Einstein) believed to originate from the continuum of space and time, instead of from physical objects.
\end{abstract}

Keywords: empty-space paradigm, micro-quanta paradigm, Doppler functions in M-Q Compton's collisions, momentum of particles, mass origin theory, relativistic dynamics in M-Q paradigm.

Revolutionary changes involve discoveries that cannot be accommodated within the concepts in use before they were made (Thomas S. Khun)

\section{Introduction}

Special Relativity arose in 1905 when a young scientist, Albert Einstein, gave an intuitive physical interpretation of the strange effects of "time dilation and length contraction" arising from the coordinate transformation of moving particles, firstly described by H.A. Lorentz, which attributed these effects to an unknown static medium (aether) filling the space. Successive experiments did not give certain indications about the aether. On this problem intervened several mathematicians and scientists of late XIX century (J.P Auffray, 2007), but their contributions were not always concordant. In 1905 Einstein assumed, rather arbitrarily, that space is empty and postulated the constancy of the light speed in any reference frame. Such simple postulates were sufficient to obtain the dynamical equations of the Relativistic Mechanics. But this exploit implied the renounce to know the physical nature of an unknown substance filling the space. General Relativity assumes that space is empty and its 
metrics is modified by the presence of masses (like in the action at a distance). However in contrast with this primitive postulate, the late Einstein believed that the gravitational interaction transmits through space with velocity c. This gives an idea of the amplitude of his mind. The criticism about some strange effects of Special Relativity (due to its kinematic origin) gave rise to several well known physical paradoxes (the Twin paradox, Ladder paradox, Bell's paradox, etc.) and even to claims of logical absurdity. No other physical theory so much attracted the interest of philosophers: an unquestionable sign that some relativistic concepts can hardly be expressed with unambiguous physical terms. However these doubts and uncertainties did not prevent the theory from obtaining empirical confirmations throughout the XX century. Many scientists attend every two years, initially in London up to 1995 and presently in Moscow, the PIRT Conferences (Physical Interpretation of Relativity Theory). This testifies that the problem of giving Special Relativity (SRT) a physical basis is still in the minds of physicists. Soon after 1905 Einstein introduced the concept of the modified metric of empty-space into the General theory of relativity which describes the gravitational phenomena through the so-called "curvature" of the empty-space. The modified metric hypothesis has been extensively applied giving results in acceptable agreement with the classical astronomic observations. But the recent enlargement of the observational universe put troubles with the relativistic cosmological models, which have been disproved by the acceleration of the far universe observed through the measure of distant Supernovae Ia (Riess, 1998, Perlmutter, 1999) in contrast with the deceleration required by the Newton's "universal constant G" assumed also in General relativity. This revolutionary discovery is discussed in par. 3 .

\section{Considerations about the New Physical Paradigms}

The most significant words on these physical "tools" have been expressed by Thomas S. Kuhn: "The overwhelming majority of scientific advance is of normal cumulative sort. Revolutionary changes are different and far more problematic. They involve discoveries that cannot be accommodated within the concepts in use before they were made. In order to make or to assimilate such a discovery one must alter the way one thinks about and describes some range of natural phenomena. The discovery of Newton's second law of motion is of this sort. The concepts of force and mass deployed in that law differed from those in use before the law was introduced and the law itself is essential to their definition". Most of Kuhn's epistemological thought may be found in the book "The Structure of Scientific Revolutions" (Kuhn, 1962) and in "The road since Structure. Philosophical Essays 1970-1993" (Kuhn, 2000). When within the current physical paradigms some problems arise that appear incompatible with the most firm and certain bases (Principles of physics), then it is hardly possible to find a solution utilising the tools of the current paradigms. An attempt carried out in the last half century was the idea of starting from the most famous theories of the XX century, i.e. General Relativity and Quantum Mechanics, to build a theory of the gravitational interaction incorporating both quantum of energy and the relativistic equations of motion. Unfortunately that attempt has not given the expected results. The reason is that between the paradigms of current physics there are some concepts that obstructs the solution.

It may appear odd to open the discussion on Special relativity beginning from the Quantum gravity, but the two arguments are tightly linked. Those who are interested to discuss the postulates of S.R.T. may jump to par.6. Now is opportune to give some premises on the physical tools (i.e. the paradigms) we use to understand the nature. Any new paradigm has to fulfil the Principles of physics, but the final proof comes from plane explanations of some previously unsolved phenomena. The paradigm of Micro-quanta has been extensively adopted (Michelini, 2011) to explain some phenomena that remained unexplained within the conceptual frame of current physics. Between these we recall three phenomena which show a thermal emission from the solar planets and their satellites many times higher than should pertain to inert celestial bodies cooling in space:

1) the excess infrared emission from internal power of the giant solar planets,

2) the Earth's frequent earthquakes, due to high thermal stresses on the solid crust, denounce an escaping thermal power much higher than the classical heat flow calculation,

3) the origin of the temperature cycles of Glacial Eras and Dansgaard-Oeschger events recorded on the ice-cores drilled in Greenland and Antarctica, still needs an explanation.

Let's recall briefly that the physical origin of these three phenomena is the M-Q gravitational power. Analysing the interaction between matter and micro-quanta, a Gravitational power arises that involves (Michelini, 2010a) all celestial masses, comprised small planets. For instance, Earth is accounted for a Gravitational power about 60 times the classical calculated value (Stein, 1995) which does not take into account the numerous earthquakes that every day shake the ocean seafloor producing fractures that eject magmatic materials. This makes planets and satellites no longer inert bodies. Recent experimental evidence has been obtained through space probes toward Jupiter and Saturn, which found that some Jupiter's satellites, in particular Io and Europe (Rathbun et al., 2003) 
and the Saturn's small satellite Enceladus (482 km diametre) show consistent volcanic activities that are unexpected in small inert bodies cooling in space probably from some billion years.

From the theoretical side, there are some conceptual knots that make difficult the elaboration of new theories aimed to give better descriptions of some phenomena. Some of these knots have found plain explanation through the M-Q paradigm (Michelini, 2010b):

1) the misconception of Newton's "gravitational mass"

2) the Quantum pushing gravity theory

3) the origin of the Mass of particles

4) the physical origin of the Inertial forces,

5) the common origin of the Strong, Weak and Gravitational fundamental forces.

\section{The M-Q paradigm Defines the Inertial Mass and the Pushing Gravitational Force}

First of all let's recall that the SRT does not extend its domain up to the Planck's scale. "One place where we do expect Special relativity to crumble is at the Planck's length. Quantum theory tell us that this scale represents a threshold below which the classical picture of spacetime disintegrates. Einstein's S.R. is part of this classical picture, so we might expect it to break down at just that point" says the physicist Lee Smolin examining the inconsistencies that arise comparing the current theories with the phenomena still not explained (Smolin, 2006). The troubles that were encountered in deriving the quantum gravity theory within the current General relativity paradigm, vanish in the quantum pushing gravity, named "pushing" to distinguish from Newton's "pulling" gravity that depends (as well as General relativity) on the old concept of "gravitational mass". Quantum pushing gravity (Michelini, 2010b) descends directly from the Micro-quanta paradigm, since two particles immersed in the micro-quanta flux experience a mutual shielding which breaks the perfect isotropy of the micro-quanta flux and gives rise to a pushing force on the two particles. Normalising the M-Q constants to the value of G, one recognises the pushing gravitational force. This fact discards the concept of "gravitational" mass. In the M-Q paradigm there is only the inertial mass which soon arises through the dynamical equation balancing the momentum exchanged (see par.9) through Compton's collisions between moving particles and micro-quanta. As described in par.5.1, the mutual shielding gives rise to a force pushing on both particles. Let's compare the Newton's force between two nucleons with the Quantum pushing gravity eq. (5.3)

$$
\mathrm{F}_{\mathrm{gr}}=\mathrm{G} \mathrm{m}_{\mathrm{n}}{ }^{2} / r^{2}=\left(\mathrm{K}_{\mathrm{n}} \sigma_{\mathrm{n}}\right) \phi_{\mathrm{o}}(2 \mathrm{Eo} / \mathrm{c})\left(\sigma_{\mathrm{n}} / 2 \pi r^{2}\right) .
$$

The measured $G$ results to depend on the constants of the micro-quanta flux. Namely: $2 \mathrm{Eo} / \mathrm{c}$ is the momentum of each bouncing quantum, $\phi_{0}$ the flux of quanta, $\sigma_{\mathrm{n}}$ the nucleon cross section (probably an electromagnetic force domain), $\mathrm{K}_{\mathrm{n}}$ is a very small non-dimensional constant indicating that incident quanta release net momentum on a very small fraction of the nucleon cross section. Rearranging eq. (3) we have $G$ in terms of the quantum constants

$$
\mathrm{G}=\mathrm{E}_{\mathrm{o}} \phi_{\mathrm{o}} \mathrm{K}_{\mathrm{n}} \mathrm{A}_{\mathrm{o}}{ }^{2} / \pi \mathrm{c}={ }_{\ni_{\mathrm{o}}} \mathrm{K}_{\mathrm{n}} \mathrm{A}_{\mathrm{o}}{ }^{2} / \pi
$$

where $\ni_{0}=E_{o} f_{0} / c$ is the M-Q energy density in space; the particle inertial mass defined by eq. (9.2) shows that the ratio $A_{o}=\sigma_{i} / m_{o i}=c^{2} / \tau_{o} \phi_{o} E_{o}$ (see eq.9.3) is constant for all particles; $\tau_{o} \approx 2 \lambda_{\mathrm{o}} / \mathrm{c}$ is the duration of the simultaneous collisions of quanta with wavelength $\lambda_{\mathrm{o}}$. Substituting $A_{o}$ one obtains the equation of $\mathrm{G}$ depending on the energy density $э_{0}$ in space

$$
\mathrm{G}=\mathrm{K}_{\mathrm{n}} \mathrm{c}^{4} / 8 \pi \lambda_{\mathrm{o}}^{2} \ni_{\mathrm{o}} .
$$

All values of the principal constants of Micro-quanta have been calculated (Michelini, 2010b) and are reported in Table 1 . The calculation require to assume the wavelength $\lambda_{\mathrm{o}}$ of micro-quanta provisionally equal to the Planck's length, while the energy density $\ni_{0}=p_{0}$ (the radiation pressure $p_{o}$ ) was calculated through eq(13.4) that gives the Strong force, as shown in par.13. So one obtains the constant $\mathrm{Kn}=1 / \mathrm{N}_{\mathrm{n}} \approx 3.94 \times 10^{-51}$. The quantity $\mathrm{N}_{\mathrm{n}}=\sigma_{\mathrm{n}} \phi \mathrm{o} \tau_{\mathrm{o}}$ $\approx 2.54 \times 10^{50}$ is the number of quanta (see eq.5.8) simultaneously colliding with a nucleon. Finally the quantum energy $\mathrm{Eo} \approx 5.9 \times 10^{-61}$ is obtained recalling that $\mathrm{Kn}=\mathrm{Eo} / \mathrm{mon} \mathrm{c}^{2}$ is the Compton's ratio in the M-Q collisions with the nucleon. This set of constants depends on the gravitational force as well as the Strong force, so the M-Q paradigm appears to rule both the micro-cosmos and the macro-cosmos. Eq.(3.2) shows that possible variations of $G$ in space and time may depend on the energy density $э_{\circ}$ of micro-quanta near large masses (galaxies and obscure supermasses). 
Table 1. The principal characteristics of micro-quanta paradigm (SI units)

\begin{tabular}{ll}
$\lambda_{\mathrm{o}} \approx 4 \times 10^{-35}$ & micro-quantum wavelength \\
$\mathrm{A}_{\mathrm{o}} \approx 4.7 \times 10^{-11}$ & constant ratio of particles (cross section / mass) \\
$\mathrm{E}_{\mathrm{o}} \approx 5.9 \times 10^{-61}$ & micro-quantum energy \\
$\phi_{\mathrm{o}} \approx 1.22 \times 10^{130}$ & micro-quanta flux \\
$\mathrm{p}_{\mathrm{o}} \approx 1.2 \times 10^{61}$ & quantum radiation pressure on particles or quantum energy density \\
$\sigma_{\mathrm{n}} \approx 7.85 \times 10^{-38}$ & inertial nucleon cross section. \\
\hline
\end{tabular}

(from Appl. Phys. Res. Vol.2, Nov. 2010)

\subsection{Cosmology: the $M-Q$ Variation of $G$ Versus the Observations of the Accelerating Universe}

Since the mutual shielding of particles gives rise to the newtonian pushing gravitation, the validity of $\mathrm{G}$ as defined by eq. (3.2) is confined within a universe of radius less than d, i.e. the mean free path due to mutual scattering in space of micro-quanta, which may be estimated of the order of $10^{25} \mathrm{~m}$. Let's recall that the mean free path of photon scattering (i.e. electromagnetic quanta) is as large as the visible universe, which can be clearly observed thanks to the negligible mutual scattering between photons. If the mutual scattering of photons were much higher, we would not see distinctly the distant galaxies. Beyond the distance $d$ the micro-quanta mutual scattering is prevalent and the celestial masses cannot feel the gravitation force of very distant galaxies.

Let's describe the variation of $\mathrm{G}(r)$ in M-Q cosmology, that is at distances larger than $\mathrm{d}$. In this case the mutual shielding of galaxies reduces and becomes negligible because the micro-quanta received from particles of each galaxy has undergone several mutual collisions along the joining line. In this way the missing beam, which originates the pushing gravitation (par.5) is replaced and the gravitation between very distant galaxies shows $\mathrm{G}(r)$ tending exponentially to zero. In this description, galaxies at distance less than $\mathrm{d}$ (near-universe) are decelerating due to the newtonian-like gravitation, whereas very far galaxies appear to be (by contrast) accelerating due to the vanishing $\mathrm{G}(r)$.

This description of the universe appears to interpret the recent observations of Ia supernovae, acting as "standard candles" in the universe (Riess 1998, Perlmutter 1999, Perlmutter-Schmidt 2003), that gave the authors the Nobel prize for Physics 2011. The work is based on the method of calculating the real distance of far galaxies, which differs from the distance arbitrarily calculated assuming that the measured redshift depends on the Hubble's law tailored on the expanding model of the universe. The M-Q description of cosmology introduces a variation of $\mathrm{G}(r)$ in space and time similar to the observed description (Riess et al. 2004). In this paper Riess wrote in the conclusions: «Utilising a simple kinematic description of the magnitude-redshift data, we find that the supernovae SNe Ia favor observed acceleration at large and past deceleration with $99,2 \%$ confidence level. [...] The best-fit redshift of the transition between these kinematic phases is $z=0.46 \pm 0.13 »$. In other words, the measurements show evidence for known deceleration in "near" universe and suggest constrains on "dark energy" evolution producing acceleration at distances greater than $z=0.46$. Let's notice that "dark energy" is a concept introduced in the GR cosmological models just to explain the unexpected acceleration of far galaxies. The link between "dark energy" and $M-Q$ energy density will appear clear.

\subsection{The variation of $G$ in Preceding Cosmological Theories}

The M-Q paradigm cancels the old Newton's concept of "universal gravitation" and explains also the failure of the GR cosmological models against the recent cosmological observations.

The hypothesis of a time-varying $\mathrm{G}$ has been studied in the past on heuristic basis through the "Large number hypothesis" (Dirac, 1938 \& 1978). During the Second world war, Einstein and his assistant at Princeton Advanced Studies, P. Bergmann, started working on a scalar field linked to time-varying G. However Einstein did not pursue this work. Subsequently in 1961 that idea was renewed in the same Institute by R. Dicke and his fellow C. Brans. They published the scalar-tensor theory (Brans-Dicke, 1961) where the scalar field was introduced in GR after generalising this theory, which does not accept the idea that G may depend on the distribution of masses in the universe. In the Einstein's theory the gravitational field can be transformed away by assuming, at least locally, an accelerated system of reference, for instance a freely falling laboratory. This "weak" Equivalence principle accepted by GR does not realise the full Principle that requires everywhere the Equivalence of the inertial and gravitational forces. However the scalar-tensor theory required to replace the undetermined $\mathrm{G}$ by another quantity $\omega$, to which the cosmologists set their limits. At present the results show (Brans, 2010) that ST theories are practically indistinguishable from the GR results in the solar system. In the 
same paper Carl Brans pointed out that a fundamental open question of physics is that of establishing a Quantum gravity theory that should reconcile the concepts of GR with those of Quantum theory.

\section{Effects of the "Gravitational Mass" Misconception}

Let's notice that in the M-Q paradigm the Equivalence principle (verified empirically with accuracy up to $10^{-12}$ ) is fully realised. Since the "gravitational" mass does not exist as source of the gravitational field, the Equivalence principle becomes a trivial identity. The "gravitational" mass $i s$ the inertial mass which, immersed in the M-Q flux, generates the gravitational field.

From the theoretical side, GRT theory contains - in a more elaborated way than Newton's theory - a physical misconception, that is the gravitational mass is assumed to be the source of the gravitational field. It has also been found that Newton's force does not verify the Principle of energy conservation (Michelini, 2010), giving the gravitational mass the (erroneous) possibility of originating an infinite energy through star collapse. This indicates the gravitational mass as the cause of the unlimited gravitational collapse, a theoretical description of collapsing stars shrinking up to material points ("black holes"). These objects, somewhat popular in the past due to their enormous gravity that prevents escaping of photons, cannot be observed in absence of electromagnetic signals. Or the material-point stars do not exist at all? Astronomers observed massive small stars (neutron stars) with very low luminosity which are remains of supernovae. These high luminosity explosions of the outer star mass, show simultaneously the core collapse up to the size of the neutron stars, that are high density bodies with mass somewhat larger than the solar mass and size of the order of Earth. No black holes. This means that theories assuming the "gravitational" mass are misleading and some their predictions may be wrong.

In the M-Q paradigm the formation of "black holes" is prevented because the gravitational pressure on matter under contraction cannot exceed a little fraction of the radiation pressure $p_{0}$ of micro-quanta (see par. 5) upon very close particles in very high density cores. Recently some astronomers (Schoedel et al., 2002) have found that in the centre of our Galaxy there are stars orbiting in 15.2 years around an obscure supermass of about 3 million times the solar mass. The relative small size of this supermass prevents enough light may reach our telescopes. But astrophysicists did not use the scheme of the unlimited collapse (black hole). They think that such enormous superbody formed through gravitational mass accretion during a time comparable to the so-called life of universe.

Under the influence of the Mach's principle, the young Einstein introduced the modified-metric of empty-space where stars and planets modify the metric of curved space in their neighbourhood, whereas all distant masses of the universe are supposed to modify the metric of the flat-space giving rise to the inertial forces. Bodies move along geodetics of space without exchanging forces between masses. There are no interaction waves in space. This anomaly excluded GR gravitation from the Unification theories of the fundamental forces (Standard model). However we must acknowledge that Einstein in his late years was altogether the man which, in its Reply to the criticism of a book contributed by many scientists (L.Schilpp, 1949), made some fundamental remarks aimed to renewing the concept of fields of force. He correctly observed that the development of the classical field theory was unsatisfying: "The Maxwell theory has remained a torso [...] Analogously the General theory of relativity furnished a field theory of gravitation, but no theory of the field-creating masses". In other words, the late Einstein showed scepticism about the concept of field-creating masses (i.e. "gravitational" masses) that he assumed in GR at the beginning.

The research towards the quantum theory of gravitation was conceptually open. The derivation of quantum gravity from GR often forgets that GR doesn't rely on pure logic, but is a semi-empirical theory incorporating the "gravitational" mass and assuming the Newton's hypothesis that $G$ is constant in time and space. This made particularly hard this way to the Quantum gravity.

\section{Quantum Pushing Gravity and the Observation of the "Gravitational" Waves}

Presently nobody agrees with Newton's action at a distance between "gravitational" masses. It appears more reliable to introduce a quantum gravitational force supported by proper interaction waves. The Micro-quanta paradigm assumes that the gravitational pushing force is transmitted by micro-waves travelling with speed vo. In 1905 Einstein assumed correctly that the velocity of light (electromagnetic waves) was the maximum attainable by particles in the void space under the action of electromagnetic waves travelling with velocity $c$. To day there are other fundamental interactions (Gravitational, Strong and Electroweak forces) with proper waves. The M-Q paradigm introduces the fifth fundamental type of forces, i.e. Inertial ones, up to now irrationally excluded. The Inertial / Gravitational and the Strong / Weak interactions are assumed to propagate through Micro-quanta with proper speed vo, so the particle momentum (eq.9.1) is given by $\mathbf{p}_{i}=m_{\mathrm{o} i} \mathbf{v} /\left[1-\left(\mathrm{v}^{2} / \mathrm{v}_{\mathrm{o}}{ }^{2}\right)\right]^{1 / 2}$. 
The velocity vo may be a bit higher than $c$ to take into account waves showing non-zero rest-mass $m_{\mathrm{o}}$. for a possible dependence of the energy $E=m_{0} c^{2} /\left[1-\left(\mathrm{v}^{2} / \mathrm{v}_{\mathrm{o}}{ }^{2}\right)\right]^{1 / 2}$ on the velocity. This happens in the case of neutrinos which at low energies show a velocity equal to that of photons. However this distinction appears to be conceptual, because in par.13 it is shown that even for high-energy neutrinos $(50 \mathrm{Gev})$ the difference $\left|\mathrm{vo}_{\mathrm{o}}-\mathrm{c}\right|$ is lower than the accuracy of the measured speed $c$. The M-Q wavelength $\lambda_{\mathrm{o}}$ is of the order of Planck's length and also the energy $E_{o} \approx 5.9 \times 10^{-61}$ is very small, since the action constant is very small.

Several experiments of detecting the "supposed" GR gravitational waves coming from far astrophysical phenomena showed up to now null results, which have put in doubt the existence of gravitational waves. The non-existence, however, repels to the physicist's conviction. Where is the misconception? The (supposed) GR waves are a theoretical "forcing", since the interactions are due to metric-deformation of empty-space. "No motion of bodies produces GR gravitational waves" says correctly (Loinger 2007) the orthodox relativist. As a consequence, any future experiment to observe micro-quanta (i.e. the gravitational waves) has to agree not necessarily with far astrophysical phenomena, but with local phenomena linked to strong inertial forces. In the M-Q paradigm the mass soon arises through the dynamical equation (par.12) balancing the momentum exchanged through Compton's collisions between moving particles and micro-quanta. Let's recall that Special relativity was legitimate by a dynamical test of Compton's scattering with X-rays only in 1922. Even after that experiment, SRT was object of a tentative to deny the quantum nature of light by physicists such as Bohr, Kramers and Slater, according to which the light showed the undulatory nature of Maxwell's waves travelling in the static aether. We have to recall that these great physicists did not believe that actions could transmit through void space without proper waves. I think that Einstein was convinced in 1905 to introduce the (arbitrary) postulate of empty-space principally by the reason to preserve the quantised nature of light, which the supporters of aether would have denied. In the M-Q paradigm the micro-quanta transmit both inertial and gravitational interaction between masses. Let's characterise the M-Q energy density

$$
\ni_{\mathrm{o}}=\phi_{\mathrm{o}} E_{\mathrm{o}} / c
$$

which exerts the M-Q isotropic radiation pressure $p_{\mathrm{o}}=\phi_{\mathrm{o}} E_{\mathrm{o}} / c$ on the scattering particles. This energy dnsity shows (Michelini, 2010) very high values of the order of $10^{61} \mathrm{~J} / \mathrm{m}^{3}$, so the physical space has an enormous energy reservoir for acting on particles through very high inertial forces. This is just what happens in the high-energy physics through collisions of accelerated particles. The micro-quanta density in space is $n_{\mathrm{o}}=\phi_{\mathrm{o}} / c$ $\approx 10^{121} / \mathrm{m}^{3}$ that corresponds to an average distance of about $10^{-40}$ metres between adjacent micro-quanta. This may appear incompatible with the micro-quanta wavelength $\lambda_{0}$ of the order of $4 \times 10^{-35} \mathrm{~m}$ (Planck's length). However it is known that the mutual cross section between photons is much less than their squared wavelength, as shown by the clear observation of the high-flux photons, emitted from far supernovae and luminous galaxies, which travel across the whole universe without showing appreciable mutual scattering. Analogously the micro-quanta do interfere between them only at very large distances. This means also that the simultaneous collisions of $\mathrm{N} \approx 10^{50}$ micro-quanta upon a nucleon happen independently on each other.

\subsection{Fundamentals of Quantum Pushing Gravity}

In the pushing gravity theory (Michelini, 2010) it was found a small fraction $\Delta \sigma$ of each particle cross-section which is forbidden (by the quantum reflection law) to quanta colliding between two particles

$$
\Delta \sigma=\mathrm{K}_{\mathrm{n}}^{1 / 2} \sigma_{\mathrm{n}}
$$

where the particles are assumed to be nucleons which constitute $99,946 \%$ of the neutral masses. The small area $\Delta \sigma$ placed on each cross section is centred on the line joining both particles. This determines a missing beam $\psi(\mathrm{r})=\Delta \sigma \phi_{0} \gamma(\mathrm{r})$ where $\gamma(r)=\Delta \sigma / 4 \pi r^{2}$ is the solid angle within which the optical scattering law forbids the passage of quanta between two particles at distance $r$. The missing beam due to the mutual shielding of two nucleons shows the strength given in brackets (calculated with the M-Q constants of Table1)

$$
\psi(r)=\Delta \sigma^{2} \phi_{\mathrm{o}} / 2 \pi r^{2}=\mathrm{K}_{\mathrm{n}} \sigma_{\mathrm{n}} \phi_{\mathrm{o}}\left(\sigma / 2 \pi r^{2}\right) \quad\left[3.76 \times 10^{42}\left(\sigma / 4 \pi r^{2}\right) \text { quanta } / \mathrm{sec}\right] .
$$

Any isolated free particle does not receive forces from collisions with the M-Q isotropic flux. The missing beam breaks the isotropy and generates a force between the two particles. In fact on the external side of each particle there is a beam $\square \psi(r)$ (opposite to the missing beam) which discharges the momentum ( $2 \mathrm{Eo} / \mathrm{c})$ of bouncing quanta giving rise to the pushing force

$$
\mathrm{F}=\left(2 \mathrm{E}_{\mathrm{o}} / \mathrm{c}\right) \psi(r)=\mathrm{K}_{\mathrm{n}} \sigma_{\mathrm{n}} \phi_{\mathrm{o}}\left(2 \mathrm{E}_{\mathrm{o}} / \mathrm{c}\right)\left(\sigma_{\mathrm{n}} / 2 \pi r^{2}\right)
$$

which, recalling the M-Q radiation pressure $p_{0}=\phi_{0} \mathrm{E}_{\mathrm{o}} / c$ of bouncing quanta pushing on both the small $\Delta \sigma$, can be put in the canonical form 


$$
\mathrm{F}=\mathrm{K}_{\mathrm{n}} \sigma_{\mathrm{n}} p_{\mathrm{o}}\left(\sigma_{\mathrm{n}} / 2 \pi r^{2}\right)
$$

We want to show that the gravitational force may be recognised through the property that classify gravity as the weakest force in nature. Let's rearrange eq.(5.3) so each quantum force $\left(2 \mathrm{E}_{\mathrm{o}} / \mathrm{c} \tau_{\mathrm{o}}\right)$ appears as the momentum released by 1 quantum $(2 \mathrm{Eo} / \mathrm{c})$ in the time $\tau_{\mathrm{o}}$

$$
\mathrm{F}=\left(\mathrm{K}_{\mathrm{n}} \sigma_{\mathrm{n}} \phi_{\mathrm{o}} \tau_{\mathrm{o}}\right)\left(2 \mathrm{Eo} / \mathrm{c} \tau_{\mathrm{o}}\right)\left(\sigma_{\mathrm{n}} / 4 \pi r^{2}\right)
$$

The factor $\left(\mathrm{K}_{\mathrm{n}} \sigma_{\mathrm{n}} \phi_{\mathrm{o}} \tau_{\mathrm{o}}\right)$ is the number of quanta pushing simultaneously on the small area $\Delta \sigma$ of each particle. The smallest number of quanta incident in the time $\tau_{\mathrm{o}}$ is 1 . Putting the factor $\left(\mathrm{K}_{\mathrm{n}} \sigma_{\mathrm{n}} \phi_{\mathrm{o}} \tau_{\mathrm{o}}\right)=1$, eq. (5.6) defines the smallest force that micro-quanta exert upon two nucleons placed at a distance $r$, that is the gravitational force

$$
\mathrm{F}_{\mathrm{gr}}=\left(\mathrm{E}_{\mathrm{o}} / \lambda_{\mathrm{o}}\right)\left(\sigma_{\mathrm{n}} / 2 \pi r^{2}\right)=\mathrm{G} \mathrm{m}_{\mathrm{n}}{ }^{2} / r^{2}
$$

The condition identifying the gravitational force

$$
\left(\mathrm{K}_{\mathrm{n}} \sigma_{\mathrm{n}} \phi_{\mathrm{o}} \tau_{\mathrm{o}}\right)=1
$$

requires that $\mathrm{K}_{\mathrm{n}}=1 /\left(\sigma_{\mathrm{n}} \phi_{\mathrm{o}} \tau_{\mathrm{o}}\right)$. By definition $\left(\sigma_{\mathrm{n}} \phi_{\mathrm{o}} \tau_{\mathrm{o}}\right)=\mathrm{N}_{\mathrm{n}}$ is the number of quanta simultaneously colliding on each side of the particle, so the constant $K_{n}=1 / N_{n}$ is such that the arbitrary force $F$ of eq(5.6) becomes the pushing gravity force $\mathrm{F}_{\mathrm{gr}}$ upon two nucleons.

\section{The Einstein's Postulates of Special Relativity}

The Einstein's postulates distinguish Special Relativity from the theories of Lorentz and Poincaré. As recognised by Einstein in his late years, Lorentz elaborated from the coordinate transformation, bearing his name, a theory of the electron motion in atoms assuming the constancy of the light speed in the static aether, a medium derived from Maxwell. Also Poincaré recognised that Lorentz transformation is everywhere verified in the physical laws, but his work was particularly directed towards the problems of two and three bodies, which generate the new branch of the intrinsic instability of the planetary system (theory of chaos). In 1905 Einstein rejected the hypothesis of aether and assumed (somewhat arbitrarily) the hypothesis of empty-space, where the velocity of light is constant in any inertial frame of reference.

The postulate of empty-space implied a renounce to other physical structures of space that could have solved the problems of classical and relativistic Mechanics. Nevertheless, thanks to his intuition, Einstein postulated the constancy of the velocity of light in any inertial frame of reference. In other words: light has the same velocity whatever be the velocity of the source, but the wavelength of light depends on the velocity of the source respect to an inertial system. Evidently these conditions are unsupported because require special properties that the empty-space does not have. The M-Q paradigm has them. This explains why SR, which demonstrated theoretically the equivalence between mass and energy, gave an incomplete explanation of the principle of Equivalence between inertial and gravitational mass. Although some extraordinarily precise experiments reduced the difference between these two masses up to $10^{-12}$, GR did not recognise that the "gravitational" mass cannot be the source of the gravitational field.

At the time (1905), Einstein was interested in the explanation of the astronomical observations that denounce the influence of light velocity on the annual parallax, the secular advance of Mercury's perihelion and the bending of light rays due to the Sun gravitational field. All astronomic phenomena have been observed through telescopes and spectrometers utilising light rays as detection devices, so he adopted the mathematics of the four dimensional space-time. The aim was the explanations of the astronomic observations taking into account in the equations of paths of the celestial bodies orbiting within the space curvature, the covariance of physical laws with the Lorentz transformation. This scheme introduced in GRT the concept of the modified-metric due to gravitational masses in the empty-space. A decision fraught with serious consequences because it cancelled the physical significance of the field of forces.

Special Relativity theory showed his capacity to explain several phenomena due to the quantised nature of electromagnetic waves (photons, X-rays, Gamma-rays) and the effects shown by particles with velocities comparable to that of light. However the first great result of SRT came up from the right interpretation of the Planck's quantum concept, i.e. the photoelectric effect that gave Einstein the Nobel Prize in 1921.

Let's examine the two Einstein's postulates that operated the jump from the kinematic quantities satisfying Lorents transformation and the dynamical quantities of the relativistic equations. We start with the second postulate, the most important one. It affirms:

"As measured in any inertial frame of reference, light is always propagated in empty space with a definite velocity $\mathrm{c}$ that is not dependent on the motion of emitting bodies" 
This representation contains the following distinct assumptions:

- inertial forces have to be referred to the inertial frames of reference (defined only in a tautological way)

- space is empty (i.e. not filled by interaction waves),

- the emission of photons through excited electrons conserves energy and momentum of the system

- all emitted photons travel through space with uniform velocity c, independently of the source speed.

Excepting the third assumption, which respects the Principles of physics, the other tree assumptions generate the following questions:

- Why empty-space? This is contrary to the concept of field based on the presence of energy density of waves in space.

- Why all photons emitted by the excited electrons in empty space show the same velocity? The empty-space doesn't have means to impose on all photons exactly the same velocity.

- Why the velocity of the photons generated by a moving source does not depend on the source speed?

These questions cannot find an answer in empty-space because light isn't the carrier in space but is carried by the micro-quanta flux. Einstein guessed in 1905 that the above proposition constituted a postulate. Only in the late years Einstein assumed the conviction that all fundamentals interactions transmit through waves with velocity $\mathrm{c}$ in non-empty space.

\section{The Photon Emission as Described in the M-Q Paradigm}

To compare Special Relativity with the Micro-quanta paradigm, let's describe the same physical event within the new paradigm. Let's consider for the sake of simplicity the photon emission from a jumping atomic electron. When an excited (accelerated) electron immersed in the flux of micro-quanta is jumping from an atomic energy-state to another, it polarises a beam of incident micro-quanta (photon) giving them extra-energy through Compton's collisions. According to the conservation of energy, the electron jumping between two energy states gives the photon the energy $\mathrm{E}_{2}-\psi(\mathrm{r}) \mathrm{E}_{1}=\mathrm{h} v$. The modulated beam (photon) shows a wavelength $\lambda=\mathrm{c} \Delta \mathrm{t}$, where $\Delta \mathrm{t}=1 / \mathrm{v}=\int \mathrm{ds} / \mathrm{v} \cong 2 \pi \mathrm{r} / \mathrm{v}$ is the time the excited electron needs to complete the average orbit (radius $\mathrm{r}$ ) of its trajectory. The conservation of momentum requires that the electron, recoiling along the photon direction with velocity $\mathbf{v}_{\mathbf{r}}$, has momentum opposite to the momentum of the photon

$$
\mathrm{m}_{e}\left|\mathbf{v}_{r}\right|=h v / c=\mathrm{v} h / 2 \pi r c
$$

where the recoil velocity is $\left|\mathbf{v}_{\mathrm{r}}\right|=\Delta \mathrm{x} / \Delta \mathrm{t}=v \Delta \mathrm{x}$. The recoil length $\Delta \mathrm{x}=\left|\mathbf{v}_{\mathrm{r}}\right| / v$ can be obtained from eq(7.1)

$$
\Delta \mathrm{x}=h / \mathrm{m}_{e} c=\lambda_{e}
$$

showing that it is just the Compton's wavelength of the electron. In other words, the photon emitted by an excited electron is generated through a recoiling collision with a micro-quanta beam. Notice that in this picture the velocity of all emitted photon is the same because they are generated by "modulation" of the micro-quanta beam travelling with velocity c. Besides, the photon wavelength $\lambda$ (as well as the energy h $\nu=h c / \lambda$ ) depends - by generation mechanism - on the source speed along the photon trajectory. This gives directly the Doppler effect of the moving source on the photon frequency. In SRT the Doppler effect of the moving source is derived apart.

This M-Q picture contains only two distinct assumptions:

1) space is filled by a flux of micro-quanta moving with velocity $\mathrm{c}$ in any direction

2) an excited (jumping) electron modulates a beam of micro-quanta.

Let's notice that these assumptions give physical meaning to the Einstein's second postulate.

\section{M-Q paradigm drops the Inertial Frames of Reference}

An important feature of the M-Q paradigm is to drop the "inertial" frames of reference. Within Special Relativity the inertial frames were logical supports defined (tautologically) as "frames where free particles follow their inertial motion". In practice the astronomers know that frames at rest respect to the large masses of the universe are good inertial frames. In M-Q paradigm there is no need of inertial frames because each particle "measures" its velocity respect to the micro-quanta incident forward and behind the particle, which assign the particle the momentum through Compton's scattering. We may know the absolute velocity of particles measuring its energy or momentum. In other words the fundamental properties of particles, such as mass, momentum and energy, materialise (see par. 9) through continuous collisions with micro-quanta. In this way the M-Q paradigm sets free the theorists from unnecessary relations with the inertial reference frames. 
To this point it appears immediately clear the first postulate of Special Relativity (called the Relativity principle) stating that within different inertial frames of reference, satisfying the Lorentz transformation of co-ordinates, the equations describing fundamental laws of physics remain formally invariant.

In M-Q paradigm the invariance of laws depends simply on the fact that particles collide with the uniform flux of micro-quanta, showing everywhere the right energy and momentum.

\section{Micro-quanta Generates the Mass and Momentum of Particles}

Adopting the same formal procedure of the subsequent par.10, it may be shown that the simultaneous Compton's collisions of the micro-quanta bouncing forwards and behind on the cross section $\sigma_{i}$ of a free particle, impose to it, adopting provisionally the usual relativistic Doppler functions, the momentum

$$
\mathbf{p}_{\mathrm{i}}=\left(\sigma_{\mathrm{i}} \tau_{\mathrm{o}} \phi_{\mathrm{o}}\right)\left(\mathrm{E}_{\mathrm{o}} / \mathrm{v}_{\mathrm{o}}^{2}\right) \mathbf{v} /\left[1-\left(\mathrm{v}^{2} / \mathrm{v}_{\mathrm{o}}^{2}\right)\right]^{1 / 2} .
$$

where $\mathbf{p}_{\mathrm{i}}$ is the sum of the momentum given up by $\mathrm{N}_{\mathrm{i}}=\left(\sigma_{\mathrm{i}} \tau_{\mathrm{o}} \phi_{\mathrm{o}}\right)$ colliding quanta. This is correct since in par.5 it has been shown that the simultaneous collisions of $\mathrm{N}_{\mathrm{i}} \approx 10^{50}$ micro-quanta upon a nucleon happen independently from each other. The quantum velocity $\mathrm{v}_{0}$, introduced in par.5 for special waves with non zero rest-mass, does not practically differs from $\mathrm{c}$ as shown in par.14. So eq(9.1) equals the S.R. particle momentum when we define the rest-mass

$$
m_{o i}=\left(\sigma_{\mathrm{i}} \tau_{\mathrm{o}} \phi_{\mathrm{o}}\right) \mathrm{E}_{\mathrm{o}} / \mathrm{c}^{2}=\mathrm{Ni}_{\mathrm{i}} \mathrm{E}_{\mathrm{o}} / \mathrm{c}^{2}
$$

where $\mathrm{N}_{\mathrm{i}}$ is the number of micro-quanta simultaneously bouncing on the cross section $\sigma_{\mathrm{i}}$ during the collisions time $\tau_{\mathrm{o}}=2 \lambda_{\mathrm{o}} / \mathrm{v}_{\mathrm{o}}$ related to the quantum wavelength $\lambda_{\mathrm{o}}$.

This result shows that the mass of particles originates from the simultaneous collisions of micro-quanta upon a scattering cross section representing a physical domain, probably of electromagnetic nature. From eq(9.1) the inertial mass of particles results to be dependent on velocity

$$
m_{i}(\mathrm{v})=m_{o i} /\left[1-\left(\mathrm{v}^{2} / \mathrm{c}^{2}\right)\right]^{1 / 2}
$$

For any particle the ratio between cross section and mass, results from eq. (9.2) to be constant

$$
\mathrm{A}_{\mathrm{o}}=\sigma_{\mathrm{i}}(\mathrm{v}) / \mathrm{mi}(\mathrm{v})=\mathrm{c}^{2} / \tau_{\mathrm{o}} \phi_{\mathrm{o}} E_{\mathrm{o}}
$$

so the particle cross section $\sigma_{\mathrm{i}}(\mathrm{v})=\mathrm{A}_{\mathrm{o}} \mathrm{m}_{\mathrm{oi}} /\left[1-\left(\mathrm{v}^{2} / \mathrm{c}^{2}\right)\right]^{1 / 2}$ is always proportional to the particle mass, which is physically supported by the particle cross section. The last one is a measure of the physical domain which scatters the micro-quanta. This completely reverses the old hypothesis that masses generate the gravitational force through the so-called gravitons. In fact micro-quanta generate both the inertial mass of particles (eq.9.2) and the gravitational force (par.5.3) between particles.

\subsection{The Origin of the Inertial Mass}

The current hypothesis of the high-mass Higg's boson arising from collapse of the quantum energy contained in a small volume of space, is possible in principle, but appears to be unnecessary when the masses of elementary particles originate, one by one, from the energy of micro-quanta incident on each cross section. This collision process creates masses of stable particles. But it reasonably acts also in dynamic situations when new particles originate from high-energy collisions.

The M-Q generation of masses puts the following fundamental question: Where the micro-quanta come from? It's excluded they are produced by "gravitational" masses. Probably micro-quanta ever existed together with the particle masses. From the standpoint of phenomenology, micro-quanta may be observed through the fields, detectable in space, of the fundamental forces (depending on the distance) as well as the inertial forces (depending on the acceleration). Strangely, these ones are not considered "fundamental forces" in the current Standard Model.

\section{Balance of the Compton's Collisions under General Principles of Physics}

Because some possible doubt remains in deriving eq.(9.1) about the adoption of the Doppler function propose in Special relativity (through kinematic reasoning), in this paragraph the momentum of particles, imposed by the Compton's collisions with micro-quanta, is derived under general principles. The M-Q paradigm requires to derive the momentum of a free particle without making hypothesis about the Doppler functions depending on the relative velocity between particle and incident micro-quanta. This relative velocity must not be defined by "ad hoc" expressions to sum the velocities respect to an inertial frame that were derived in S.R. on kinematic grounds. The particle velocity descends directly from the dynamical interaction of particles with micro-quanta under the laws of conservation of Energy and Momentum expressed by the Compton's simultaneous collisions. 
Like the usual quanta, the micro-quantum energy is given by $E_{o}=h_{o} v_{o}$ where $v_{0}$ is the quantum frequency and $h_{o}$ is the proper action constant. Let's put $\mathrm{g}_{\mathrm{b}}(\mathrm{v})$ and $\mathrm{g}_{\mathrm{f}}(\mathrm{v})$ the unknown Doppler functions of the $\mathrm{N}_{\mathrm{b}}$ quanta incident behind with the $N_{f}$ quanta incident forward the particle, so the momenta $q_{b}=h_{o} v_{b} / c$ and $q_{f}=h_{o} v_{f} / c$ given up by quanta bouncing on each side depends on the Doppler frequencies $v_{\mathrm{b}}(\mathrm{v})=\mathrm{g}_{\mathrm{b}}(\mathrm{v}) v_{\mathrm{o}}$ and $v_{\mathrm{f}}(\mathrm{v})=\mathrm{g}_{\mathrm{f}}(\mathrm{v}) v_{\mathrm{o}}$. The momentum of the particle originates from the momentum that the simultaneous Compton's collisions assign to the particle. We recall the equation which gave $\mathbf{p}_{i}$ under general assumptions in a preceding paper (Michelini, 2010)

$$
\left|\mathbf{p}_{i}\right|=\mathrm{N}_{\mathrm{b}} E_{\mathrm{b}} / c-\mathrm{N}_{\mathrm{f}} E_{\mathrm{f}} / c=\left(h_{o} / c\right) \sigma_{\mathrm{i}}\left(\tau_{\mathrm{b}} \phi_{\mathrm{b}} v_{\mathrm{b}}-\tau_{\mathrm{f}} \phi_{\mathrm{f}} v_{\mathrm{f}}\right)
$$

where $\mathrm{N}_{j}=\sigma_{\mathrm{i}} \phi_{\mathrm{j}} \tau_{\mathrm{j}}(j=\mathrm{b}, \mathrm{f})$ is the number of micro-quanta simultaneously colliding behind and forwards the particle in the time $\tau_{o}$. Substituting by definition $\tau_{j} v_{j}=1$, one gets

$$
\left|\mathbf{p}_{i}\right|=\sigma_{i}\left(h_{o} / c\right)\left(\phi_{\mathrm{b}}-\phi_{\mathrm{f}}\right)
$$

The fluxes $\phi_{\mathrm{b}}$ and $\phi_{\mathrm{f}}$ can be derived from the conservation of energy density between the incident micro-quanta and the energy density $э_{0}=\phi_{0} E_{0} / c$ in free space. Since a free particle with constant velocity does not suffer any force from the micro-quanta flux, this requires the equality between the energy density of quanta incident behind $\left(\ni_{\mathrm{b}}\right)$ and forward $\left(\ni_{\mathrm{f}}\right)$

$$
\ni_{\mathrm{o}} / 2=\phi_{\mathrm{o}} \mathrm{E}_{\mathrm{o}} / 2 c=\ni_{\mathrm{b}}=h_{\mathrm{o}} \phi_{\mathrm{b}} v_{\mathrm{b}} / c=\ni_{\mathrm{f}}=h_{\mathrm{o}} \phi_{\mathrm{f}} v_{\mathrm{f}} / c .
$$

When the frequency $v_{\mathrm{f}}$ of quanta incident forward increases by Doppler during the interaction, the corresponding flux $\phi_{\mathrm{f}}=\phi_{\mathrm{o}} v_{\mathrm{o}} / v_{\mathrm{f}}$ decreases. Substituting $\phi_{\mathrm{b}}$ and $\phi_{\mathrm{f}}$ in eq. (10.2) one gets

$$
\left|\mathbf{p}_{i}\right|=\sigma_{\mathrm{i}} \phi_{\mathrm{o}}\left(h_{\mathrm{o}} / 2 c\right)\left[v_{\mathrm{o}} / v_{\mathrm{b}}-v_{\mathrm{o}} / v_{\mathrm{f}}\right]=\sigma_{\mathrm{i}} \phi_{\mathrm{o}} \tau_{o}\left(\mathrm{E}_{\mathrm{o}} / 2 \mathrm{c}\right)\left[1 / \mathrm{g}_{\mathrm{b}}-1 / \mathrm{g}_{\mathrm{f}}\right] \text {. }
$$

Now we write the balance between this quantity and the particle momentum $|\mathbf{p}|=\mathrm{m}_{\mathrm{o}} \mathrm{v} / \beta_{\mathrm{x}}$ where $\beta_{\mathrm{x}}$ is an unknown function depending on the velocity ratio $(\mathrm{v} / \mathrm{c})$

$$
\left|\mathbf{p}_{i}\right|=\mathrm{N}_{\mathrm{i}}\left(\mathrm{E}_{\mathrm{o}} / 2 c\right) \quad\left[1 / \mathrm{g}_{\mathrm{b}}-1 / \mathrm{g}_{\mathrm{f}}\right]=m_{\mathrm{oi}} \mathrm{v} / \beta_{\mathrm{x}} .
$$

On the other hand, the energy $\mathcal{E}_{i}$ originating from the sum of the simultaneous Compton's collisions must be equal to the energy $m_{\mathrm{oi}} \mathrm{c}^{2} / \beta_{\mathrm{x}}$ of the particle

$$
\mathrm{E}_{i}=\mathrm{N}_{\mathrm{i}}\left(\mathrm{E}_{\mathrm{o}} / 2\right)\left[\left(1 / \mathrm{g}_{\mathrm{b}}+1 / \mathrm{g}_{\mathrm{f}}\right]=m_{\mathrm{oi}} c^{2} / \beta_{\mathrm{x}} .\right.
$$

Summing and subtracting eq. (10.5) and eq. (10.6) one obtains the Doppler functions

$$
\begin{aligned}
& \mathrm{g}_{\mathrm{b}}(\mathrm{v})=m_{\mathrm{oi}} c^{2} /\left(\mathcal{E}_{i}+c \mathrm{p}_{i}\right)=\beta_{\mathrm{x}} /(1+\mathrm{v} / c) \\
& \mathrm{g}_{\mathrm{f}}(\mathrm{v})=m_{\mathrm{oi}} c^{2} /\left(\mathcal{G}_{i}-c \mathrm{p}_{i}\right)=\beta_{\mathrm{x}} /(1-\mathrm{v} / c)
\end{aligned}
$$

which depend on the energy and momentum of the particle. Multiplying side by side we obtain the equation

$$
\mathrm{g}_{\mathrm{f}}(\mathrm{v}) \mathrm{g}_{\mathrm{b}}(\mathrm{v})=m_{\mathrm{oi}}{ }^{2} \mathrm{c}^{4} /\left(\mathcal{G}_{\mathrm{i}}^{2}-\mathrm{c}^{2} \mathrm{p}_{\mathrm{i}}^{2}\right) \text {. }
$$

If we compare with the equation showing the link between energy and momentum of particles

we obtain the condition

$$
m_{\mathrm{oi}}^{2} \mathrm{c}^{4}=\mathcal{E}_{i}^{2}-\mathrm{c}^{2} \mathrm{p}_{\mathrm{i}}^{2}
$$

$$
\mathrm{g}_{\mathrm{f}}(\mathrm{v}) \mathrm{g}_{\mathrm{b}}(\mathrm{v})=1
$$

which holds in general. As a consequence, one obtains from eq.(10.7) and eq(10.8) the Doppler functions for waves incident forwards and behind any moving particle

$$
\mathrm{g}_{\mathrm{f}}(\mathrm{v})=(1+\mathrm{v} / \mathrm{c})^{1 / 2} /(1-\mathrm{v} / \mathrm{c})^{1 / 2} ; \mathrm{g}_{\mathrm{b}}(\mathrm{v})=(1-\mathrm{v} / \mathrm{c})^{1 / 2} /(1+\mathrm{v} / \mathrm{c})^{1 / 2}
$$

that result equal to the SR Doppler functions.

Multiplying side by side eq. (10.7) and eq.(10.8) and comparing with eq(10.11) we obtain the parameter

$$
\beta_{x}^{2}=\left(1-v^{2} / c^{2}\right)
$$

that equals the S.R. parameter $\beta$.

\section{The Fundamental Equation of Dynamics in the M-Q Paradigm}

The inertial force on a particle depends in the classical and relativistic paradigms on a variation $\mathrm{d}(\mathrm{m} \mathbf{v})$ of momentum caused by an external force during the time $\mathrm{dt}$. In the $\mathrm{M}-\mathrm{Q}$ paradigm the inertial force comes directly from the quantum properties since it originates from the net momentum of the simultaneous Compton's collisions discharged in the unit of time by the micro-quanta incident forwards $\left(\mathrm{E}_{\mathrm{f}}\right)$ and behind $\left(\mathrm{E}_{\mathrm{b}}\right)$ the particle 


$$
\left|\boldsymbol{F}_{\mathrm{i}}\right|=\sigma_{\mathrm{i}} \phi_{\mathrm{b}}\left(\mathrm{E}_{\mathrm{b}} / \mathrm{c}\right)-\sigma_{\mathrm{i}} \phi_{\mathrm{f}}\left(\mathrm{E}_{\mathrm{f}} / \mathrm{c}\right)=\sigma_{\mathrm{i}}\left(\ni_{\mathrm{b}}-\ni_{\mathrm{f}}\right) .
$$

The right-side comes immediately from substituting the energy density $\ni_{\mathrm{j}}=\phi_{\mathrm{j}} \mathrm{E}_{\mathrm{j}} / \mathrm{c}$ of micro-quanta. This equation shows that considering a free particle moving with constant and uniform velocity, the conservation (see par.10) of the M-Q energy density $\left(\ni_{b}=\ni_{f}\right)$ gives null inertial force (principle of inertia). Recalling that the energy density of micro-quanta equals their radiation pressure upon particles, eq. (11.1) may be written

$$
\left|\boldsymbol{F}_{\mathrm{i}}\right|=\sigma_{\mathrm{i}}(\mathrm{v})\left(p_{\mathrm{b}}-p_{\mathrm{f}}\right)
$$

where $p_{\mathrm{b}}, p_{\mathrm{f}}$ are the $\mathrm{M}-\mathrm{Q}$ radiation pressures behind and forwards the particle. In the M-Q paradigm the particle acts as a space domain (probably of electromagnetic nature) scattering the incident micro-quanta. Then the inertial forces depend on the net balance of the M-Q radiation pressures behind and forwards the particle.

\subsection{Why Sr Introduces the Longitudinal and Transversal Mass of Particles?}

We have now to show the difference between this fundamental equation in $\mathrm{M}-\mathrm{Q}$ paradigm and the mathematical definition of force in SR as the momentum derivative respect to time $\mathbf{F}=\mathrm{d} \mathbf{p} / \mathrm{dt}$ assuming the continuity of forces and momentum in the mathematical continuous of space and time. Incidentally this procedure gives rise to the difference between the so-called longitudinal and transversal mass of particles, which remains an open question in SRT. Let's write the mathematical definition of force in the frame of the M-Q paradigm, where the momentum $\mathbf{p}_{\mathrm{j}}=\mathrm{m}_{\mathrm{oj}} \mathbf{v} /\left[1-\left(\mathrm{v}^{2} / \mathrm{c}^{2}\right)^{1 / 2}\right]$ is the same given by SR, but the infinitesimal time $\mathrm{dt}$ is substituted by the duration time $\tau_{\mathrm{o}}$ of the simultaneous collisions. Since in the M-Q paradigm the inertial force eq.(11.2) arises from the spatial configuration of collisions which determines the speed variation $\Delta \mathbf{v}$, the actual mass (depending on Doppler, namely on velocity) is constant during the collisions. So one gets the $\mathrm{M}-\mathrm{Q}$ expression

$$
\boldsymbol{F}_{\mathrm{j}}=m_{\mathrm{oj}} \Delta\left\{\mathbf{v} /\left[1-\left(\mathrm{v}^{2} / \mathrm{c}^{2}\right)\right]^{1 / 2}\right\} / \tau_{\mathrm{o}}=\left\{m_{\mathrm{oj}} /\left[1-\left(\mathrm{v}^{2} / \mathrm{c}^{2}\right)\right]^{1 / 2}\right\} \Delta \mathbf{v} / \tau_{\mathrm{o}} .
$$

The apparent duality of the particle mass originates in SR from the pure mathematical (i.e. not physical) definition of force. Not always mathematical results have significance in physics. In this case the right-side of eq (11.3) differs from the SR inertial forces because there is no mass difference between the circular uniform motion and the strait-line accelerate motion. A difference between the SR and the M-Q inertial forces is the substitution of the finite quantities $\Delta \mathbf{v} / \tau_{0}$ ratio with the ratio of infinitesimal quantities dv/dt. Both these notations are obviously accurate due to the very little duration time $\tau_{\mathrm{o}} \approx 10^{-43} \mathrm{sec}$ of the simultaneous collisions respect to the common particle's phenomena. Anyway, in the M-Q paradigm the right definition of quantum inertial force is given by eq. (11.2).

\section{Inertial Forces Originate from the Local M-Q Energy Density}

Eq. (11.3) becomes of great importance when compared with the fundamental eq. (11.2) where the cross section is substituted by $\sigma_{\mathrm{l}}(\mathrm{v})=\mathrm{A}_{\mathrm{o}} m_{\mathrm{j}}(\mathrm{v})=\mathrm{A}_{\mathrm{o}} m_{\mathrm{oj}} /\left[\tilde{1}-\left(\mathrm{v}^{2} / \mathrm{v}_{\mathrm{o}}^{2}\right)\right]^{1 / 2}$

$$
\left|\boldsymbol{F}_{\mathrm{j}}\right|=\left\{m_{\mathrm{oj}} /\left[1-\left(\mathrm{v}^{2} / \mathrm{v}_{\mathrm{o}}^{2}\right)\right]^{1 / 2}\right\}\left|\Delta \mathrm{v} / \tau_{\mathrm{o}}\right|=\mathrm{A}_{\mathrm{o}}\left\{m_{\mathrm{oj}} /\left[1-\left(\mathrm{v}^{2} / \mathrm{v}_{\mathrm{o}}^{2}\right)\right]^{1 / 2}\right\}\left(p_{\mathrm{b}}-p_{\mathrm{f}}\right) .
$$

This equation shows that the balance $\left(p_{b}-p_{f}\right)$ of the M-Q radiation pressures behind and forwards the particle is due to the variation of its velocity during the time of simultaneous Compton's collisions

$$
\left|\Delta \mathbf{v} / \tau_{\mathrm{o}}\right|=\mathrm{A}_{\mathrm{o}}\left(p_{\mathrm{b}}-p_{\mathrm{f}}\right) .
$$

This equation is the unique physical reason why the inertial forces depend on the particle acceleration, so giving the physical basis of the Newton's law of Dynamics that remained famous for its accuracy. Substituting eq(9.3) which defines $A_{o}=c / \tau_{o} \mathrm{p}_{o}$, one obtains the simple equation

$$
\left(p_{\mathrm{b}}-p_{\mathrm{f}}\right)=p_{\mathrm{o}}|\Delta \mathbf{v} / c|
$$

which shows the net radiation pressure acting on accelerated particles is proportional to the ratio $|\Delta v / \mathrm{c}|$, that is the variation of the ratio $(\mathrm{v} / \mathrm{c})$ during the time $\tau_{o}$ of the simultaneous Compton's collisions. Multiplying and dividing the ratio $|\Delta \boldsymbol{v} / \mathrm{c}|$ by $\tau_{o}$ one obtains $\Delta \boldsymbol{v} / c=\Delta \boldsymbol{x} / 2 \lambda_{o}$, where $\Delta \boldsymbol{x}$ is the particle displacement respect to the position occupied in the uniform motion. Then eq.(12.3) may be replaced by

$$
\left(p_{\mathrm{b}}-p_{\mathrm{f}}\right) / p_{\mathrm{o}}=\left|\Delta \mathbf{x} / 2 \lambda_{o}\right| \text {. }
$$

This particular adimensional equation is formally similar to the structure of the elasticity theory of ideal monokinetic quanta where the percent stress upon a cross section equals the percent compression/decompression on the quantum wavelength (strain). This may be explained considering that an accelerated particle $(\Delta \mathbf{v}>0)$ undergoes a forwards displacement $\Delta \mathbf{x}$ that compresses the M-Q energy density, so increasing the forwards radiation pressure $\left(p_{\mathrm{f}}>p_{\mathrm{o}}\right)$ and simultaneously decreasing the behind radiation pressure $\left(p_{\mathrm{b}}<p_{\mathrm{o}}\right)$. In this way the particle feels the inertial force defined by eq.(11.2) that counterbalances the accelerating external force. 
Viceversa a decelerating particle $(\Delta \mathbf{v}<0)$ feels an inertial force that counterbalances the decelerating external force and tends to maintain the initial kinetic energy.

To resume, the M-Q flux does not interact with particles in uniform motion with constant velocity ( $\mathrm{v} / \mathrm{c}=\mathrm{const}$ ), so, $\mathrm{M}-\mathrm{Q}$ flux acts as a perfect fluid. Besides the $\mathrm{M}-\mathrm{Q}$ radiation pressure on the accelerated particle gives rise to inertial forces that always counterbalance the external forces acting on the particle.

We know that when particles travel with constant velocity (principle of inertia) the incident micro-quanta show an increasing frequency (Doppler function) during the approaching interaction and a decreasing frequency during the removing interaction, so the bouncing quanta come back to their initial frequency without exchanging energy and momentum with the particle. Conversely, a moving particle subjected to an external force, receives from the M-Q flux a counter balancing inertial force that increases (or reduces) the particle kinetic energy. This requires that quanta bouncing forwards on accelerating particles show an energy higher than the initial one, that appear as a travelling ripple (wave) of the stationary energy density. The opposite behaviour is shown by a decelerated particle. In this way some of the particle kinetic energy is brought away by the scattered micro-quanta. Might these fluctuations/waves of micro-quanta be observed?

\section{The Common Origin of the Strong, Weak and Gravitational Forces}

There is general consensus to the idea that the three fundamental drawing forces (Gravitational, Strong and Weak forces) arise from a common interaction between particles and waves travelling with the velocity of light. It is a good opportunity to verify if the M-Q paradigm supports the "unification" of these forces. As we know, the calculation of the smallest fraction of cross section $\Delta \sigma=\mathrm{K}_{\mathrm{n}}{ }^{1 / 2} \sigma_{\mathrm{n}}$ that presents the missing beam phenomenon gave rise to the Gravitational force (par.5.1). It has been searched (Michelini, 2008) if there are - on two equal particles - possible fractions of cross section $\Delta \sigma(r)$ depending on the distance, that presents the missing beam phenomenon. It was found that

$$
\Delta \sigma(r)=(\sigma / 2)\left(\sigma / 2 \pi r^{2}\right)
$$

so the missing beam (i.e. quanta that cannot play "ping pong" between two particles) results

$$
\psi(r)=\Delta \sigma(r) \phi_{\mathrm{o}} \gamma(r)=(\sigma / 4) \phi_{\mathrm{o}}\left(\sigma / 2 \pi r^{2}\right)^{3}
$$

where $\gamma(r)=\Delta \sigma(r) / 2 \pi r^{2}$ is the solid angle within which is forbidden the passage of quanta between the two particles. So the force due to the external pushing beams (opposite to the missing beam) is on both particles

$$
\Phi(r)=\psi(r)\left(2 \mathrm{E}_{\mathrm{o}} / \mathrm{c}\right)=\left(\mathrm{E}_{\mathrm{o}} \phi_{\mathrm{o}} / 2 \mathrm{c}\right) \sigma\left(\sigma / 2 \pi r^{2}\right)^{3}
$$

\subsection{The Strong force between Nucleons}

The way to define the Strong force in the M-Q paradigm is physically more understandable than the current theory proposed by the Standard Model. Substituting the M-Q radiation pressure $p_{\mathrm{o}}=\mathrm{E}_{\mathrm{o}} \phi_{\mathrm{o}} / 2 \mathrm{c}$ that exerts a pushing force on the close particle pairs, one gets between two nucleons the force

$$
\Phi(r)=\sigma_{\mathrm{n}} p_{\mathrm{o}}\left(\sigma_{\mathrm{n}} / 2 \pi r^{2}\right)^{3}
$$

that presents characteristics similar to the qualitative description of the current Strong force "The decrease is approximately as a negative exponential power of distance, though there is no simple expression known for this"(see Wikipedia en.). Substituting $\sigma_{\mathrm{n}} \approx 7.85 \times 10^{-38}$ and $p_{o} \approx 1.2 \times 10^{61}$ taken from the approximate set of constants of micro-quanta (Michelini, 2010b) reported in Table 1, this equation assumes the form (SI units)

$$
\Phi(r) \approx 1.83 \times 10^{-90} / r^{6}
$$

Let's verify if this equation - giving the first explicit definition of the drawing force between nucleons - is the so-called Strong force that holds together the atomic nuclei. The drawing force $\Phi(r)$ exceeds the electric repulsion of a pair of protons if the mutual distance is lower than about $3 \times 10^{-16}$, but the equilibrium is unstable. Actually in the real stable nuclei there are neutrons, which do not repel other nucleons, so they make stable the nuclear assemblies. In principle it could be possible to verify the unknown constant $p_{\mathrm{o}}$ for any nucleus, if one knows its internal structure. In practice the problem becomes complex because it needs to study any stable configuration of the existing nuclei. Assuming for instance that the Strong forces exerted by neutrons (concentrated in the nuclear core) and by protons (placed on the external nuclear surface) may be able to maintain a stable configuration, the stability equation of an external proton at distance $r_{\mathrm{N}}$ (nuclear radius) results to be

$$
(Z-1) \mathrm{e}^{2} / 4 \pi \varepsilon_{\mathrm{o}} r_{\mathrm{N}}^{2} \approx \Sigma_{\mathrm{i}} \cos \alpha_{\mathrm{i}} \Phi\left(x_{\mathrm{i}}\right) \approx \Sigma_{\mathrm{i}} \cos \alpha_{\mathrm{i}} \sigma_{\mathrm{n}} p_{\mathrm{o}}\left(\sigma_{\mathrm{n}} / 2 \pi x_{\mathrm{i}}^{2}\right)^{3}
$$


where the summation is extended to the nearest nucleons. The nucleon cross section is assumed to have the tabulated value $\sigma \approx 7.85 \times 10^{-38}$ and $x_{\mathrm{i}}$ is the distance of the nearest nucleons from the external proton. The value of $p_{\mathrm{o}}$ given in Table 1 is derived through check and errors.

A check of the force $\Phi(r)$ has been done (Michelini, 2008) calculating the orbit of the Deuteron constituted by a neutron-proton pair orbiting each other at a distance of about $1.65 \times 10^{-16}$.

\subsection{The Weak Force on the Electron}

The strong-force $\Phi(r)$ is commutative between two equal particles. When a particle with cross section $\sigma_{\mathrm{i}}=\left(m_{\mathrm{i}}\right.$ $\left./ m_{n}\right) \sigma_{\mathrm{n}}$ is close to a nucleon with cross section $\sigma_{\mathrm{n}}$, the force $\Phi(r)$ becomes

$$
\Psi_{\mathrm{i}, \mathrm{n}}(r)=\sigma_{\mathrm{i}} \quad p_{\mathrm{o}}\left(\sigma_{\mathrm{n}} / 2 \pi r^{2}\right)^{3}
$$

which is non-commutative and breaks the symmetry of the action-reaction principle. For instance, considering an electron $\left(\sigma_{\mathrm{e}}=\sigma_{\mathrm{p}} / 1836\right)$ closely bound to a proton, the Weak-force equals numerically the Strong-force $\Phi(r)$ divided by 1836, obtaining upon the electron the force

$$
\Psi_{\mathrm{e}, \mathrm{p}}(r) \approx 10^{-93} / r^{6} \text {. }
$$

As expected, Gravitational, Strong and Weak forces are all drawing forces due to their common origin from the mutual shielding of the Micro-quanta flux. Only exception in the Standard Model is the Electromagnetic force which can be repelling or drawing.

\section{On the Velocity of Interaction Waves}

A century ago, Einstein was entirely legitimate to assume the speed of light as the highest attainable in nature. To day the problem of the velocity of waves comes again into discussion. Besides the open question of the time delay of mysterious $\gamma$-ray bursts observed by the Fermi $\gamma$-ray Space Telescope (Naeye R., 2008), recently some different attempts to measure the velocity of high-energy neutrino beams gained the attention. However any comparison between the neutrino velocity and the light velocity should be performed across the same medium. Since light cannot travel within rocks, but we know with great accuracy its velocity in free space, also the neutrino velocity should be measured in free space on equal path. This will eliminate even the difficulty of precise measuring of the path length and elapsed time.

In the CERN-OPERA experiment the velocity of neutrinos has been calculated as ratio between the measured path length under the Earth crust and the time elapsed in the travel. Apart the practical difficulties to obtain such accurate measurements, this attempt does not give the neutrino velocity under reproducible conditions. As it has been recalled by the Nobel prize S. Glashow, the collisions that neutrinos undergo within the rocks change their energetic composition.

Travelling under rocks, the beam loses neutrinos with high initial energy (40-50 Gev) through collisions with electrons, so the detected neutrinos show lower energy (about $17 \mathrm{Gev}$ ). Anyway, during the trip the neutrino's energy (between 50 and $17 \mathrm{Gev}$ ) is much higher than the usual neutrino coming from astrophysical events. We have to take in mind that neutrinos are waves with non zero rest-mass, so their energy depend on velocity and, conversly, their velocity depend on energy.

Neutrinos of low energy received from the supernova of 1987 were detected at the same time of supernova light rays, so they show - in free space - the velocity c. If these neutrinos were travelling with the same velocity found in a first attempt by the Opera experiment, they would have arrived four years in advance of the supernova light. This showed that Opera experiment was probably affected by some troubles in the instrumental chain, as it was subsequently publicly denounced.

We are interested to point out that in the M-Q paradigm it may be correctly supposed that waves with rest-mass $m_{\mathrm{o}_{\mathrm{i}}}>$ 0 , such as neutrinos, may show velocities different than $\mathrm{c}$. In this case the energy may be correctly given by $E_{\mathrm{v}}=$ $m_{\mathrm{ov}} c^{2} / \beta$, where the parameter $\beta=\left(1-\mathrm{v}_{\mathrm{v}}{ }^{2} / \mathrm{v}_{\mathrm{o}}{ }^{2}\right)^{1 / 2}$ is referred to the micro-quanta base-velocity vo. Assuming that light velocity is a bit lower than the base-velocity, the neutrino's high-energy may depend on a neutrino velocity $\mathrm{v}_{v}$ slightly higher than $c$. However some calculations, referred to the (muonic) neutrino energy adopted at Opera experiment (about $50 \mathrm{Gev}$ ) show that to observe the effect $v_{0}>v_{v}>c$ the base-velocity $v_{o}$ should result $v_{0} / c$ $\geq 1+4.5 \times 10^{-12}$. This difference between the velocities $\mathrm{v}_{\mathrm{o}}$ and $c$ is negligible, surely lower than the error $\Delta c / c \approx 10^{-9}$ in the measurement of $c$ in free space. Thus it appears very hard to measure experimentally a difference between $v_{v}$ and $c$ not exceeding $4.5 \times 10^{-12} c$. The low-energy neutrinos showed speeds equal to $c$, as denounced by the 
Supernova observation in 1987. In practise the neutrino velocity effect $v_{v}>c$ may exists, but cannot be really measured.

\section{Beta Decay: Electron-Neutrino Does not Take Away Any “Missing” Energy}

The description of the strong-interaction forces between particles in M-Q paradigm (par.13) recalls us the reason why in $1930 \mathrm{~W}$. Pauli postulated in a letter to his colleagues the existence of a new undetectable particle (he suggested the name of neutron). The reason was to preserve in the $\beta^{-}$decay

$$
\mathrm{n}^{\mathrm{o}} \rightarrow \mathrm{p}^{+}+e^{-}+\bar{U}_{e}
$$

the conservation of energy, momentum and spin. This particle was charge-less, ideally massless, but carrying away a lot of kinetic energy subtracted to the electron. After the Chadwick's discovery in 1932 of the massive neutron, E. Fermi, who developed the first theory of the beta decay (1934) giving a basis to the future work, renamed neutrino the postulated particle to avoid confusion. History tells us that at the 1933 Solvay's Conference W. Pauli announced with some reluctance his hypothesis about the neutrino. Only in 1956 this elusive particle could be captured by Cowan and Reines, but in their experiment

$$
\mathrm{p}^{+}+\overline{\mathrm{U}}_{\mathrm{e}} \rightarrow \mathrm{n}^{\mathrm{o}}+\mathrm{e}^{+}
$$

the $\overline{\mathrm{U}}_{\mathrm{e}}$ electron anti-neutrino gives up energy to a proton instead of subtracting energy to a lepton. There is poor certainty that this neutrino is physically identical to that appearing in eq(15.1). As explained in the following, most probably the elusive neutrino of $\beta^{-}$decay was never detected because it didn't exist. The energy spectrum of the emitted electron depends on other reasons.

As a matter of fact, the characteristics of neutrinos - says a neutrinos' skilled physicist - have been not definitely established after eight decades of research (Anicin, 2005). Since the Pontecorvo's early suggestion (1958) about the neutrino "oscillations", many experiments have been done to determine the change of mass. Three kinds of neutrinos (electronic, muonic, tauonic) with different mass-energy have been proposed to explain the various experiments. However at the beginning the problem was to account only for the loss of energy conservation in $\beta^{-}$ decay. From the experimental measurements the electron kinetic energy shows a spectrum that varies between zero and $\Theta \mathrm{Mev}$, where $\Theta$ may take values from a few Kev up to a few tens of Mev for several isotope decay.

According to the classical interpretation, when the electron kinetic energy $\mathrm{E}_{\mathrm{k}, \mathrm{e}}(i)$ is less than $\Theta$ Mev, the difference is carried away by the anti-neutrino. This reasoning was incomplete. The rest-mass difference (neutron - proton - electron $)=0.78 \mathrm{Mev}\left(1.25 \times 10^{-13} \mathrm{Joule}\right)$ is much less than the kinetic energy $\Theta$, which for some decaying isotopes goes over 16.4 Mev. Then, the proper question is: There is some nucleon unknown bound-state that releases the variable energy $\mathrm{E}_{\mathrm{x}}(i)$ at each decay? In other words, the energy balance of the $\beta^{-}$ decay has to be written

$$
0.78 \mathrm{Mev}+\mathrm{E}_{\mathrm{x}}(i)=\mathrm{E}_{\mathrm{k}, \mathrm{e}}(i)
$$

So we don't have to speak of "missing" energy, but we have to find the internal mechanism that gives the (or positron) an energy $\mathrm{E}_{\mathrm{x}}(i)$ comprised between 0.78 and $\Theta \mathrm{Mev}$.

At present there is a possible explanation. The drawing force $\Psi_{\mathrm{e}, \mathrm{p}}(r)$ on the bound-electron has been explicitly given in eq(13.9). As a consequence we have to verify whether the energy of $\beta^{-}$decay equals the kinetic energy $\mathrm{E}_{\mathrm{x}}(i)$ of the bound-electron to counterbalance the weak-force $\Psi_{\mathrm{e}, \mathrm{p}}(r)$. Due to a triggering cause, the electron escapes from the bound-state. Following the scheme of eq. (15.3) let's consider the decay ${ }_{7} \mathrm{~N}^{17} \rightarrow{ }_{8} \mathrm{O}^{17}+\mathrm{e}^{-}$which shows a maximum kinetic energy of the electron $\Theta=8.7 \mathrm{Mev}=1.39 \times 10^{-12}$ Joule. By eq.(15.3) one gets the energy $\mathrm{E}_{\mathrm{x}}(i)$ $=1.27 \times 10^{-12}$ of the bound-state. Let's equate this value to the energy attained by the electron during the capture through the drawing force $\Psi_{\mathrm{e}, \mathrm{p}}(r) \approx 10^{-93} / r^{6}$, reaching an orbit of radius $r_{\mathrm{e}}$

$$
\int_{r_{e}}^{\infty} 10^{-93} d r / r^{6} \cong 10^{-93} / 5 r^{5}=1.27 \times 10^{-12}
$$

This equation gives a radius of the bound state

$$
r_{\mathrm{e}} \approx\left(10^{-93} / 6.35 \times 10^{-12}\right)^{1 / 5}=4.36 \times 10^{-17}
$$

which is congruent with the mean distance of about $(4 \div 5) \times 10^{-16}$ between two nucleons in nucleus (see par.13).

In the case of $\beta^{+}$decay the M-Q description is even more convincing. Consider for instance the interaction ${ }_{7} \mathrm{~N}^{12}$ $\rightarrow{ }_{6} \mathrm{C}^{12}+\mathrm{e}^{+}$where the decay of proton [ bound-state neutron + positron] assumes the energy balance 


$$
\left|\mathrm{p}^{+}\right|=|\mathrm{n}|+\mathrm{E}_{\mathrm{x}}(i)+\left|\mathrm{e}^{+}\right| \rightarrow|\mathrm{n}|+\left|\mathrm{e}^{+}\right|+\mathrm{E}_{\mathrm{k}, \mathrm{e}}(i)
$$

where the positron, which feels the weak-force only, shows a maximum kinetic energy $\Theta=16.4 \mathrm{Mev}$. Then the bound positron has a maximum kinetic energy $\mathrm{E}_{\mathrm{x}}(\mathrm{i})$ equal to $\Theta=16.4 \mathrm{Mev}=2.62 \times 10^{-12}$ attained during capture by the neutron drawing force

$$
\int_{\infty}^{r} 10^{-93} d r / r^{6}=10^{-93} / 5 r_{p}=2.62 \times 10^{-12}
$$

forming a bound-state of radius

$$
\mathrm{r}_{\mathrm{p}} \approx\left(10^{-93} / 1,31 \times 10^{-11}\right)^{1 / 5}=3.71 \times 10^{-17} .
$$

In the bound-states of nucleons the weak-force on the electron is higher than the electric force, which acts also on the proton. The proton does not change kinetic energy since feels a negligible (non-commutative) force $\Psi_{\mathrm{p}, \mathrm{e}}(r)$ from the electron.

The present explanation of the phenomenon suggests that the hypothesised neutrino does not exist in $\beta$ decay. However the evidences of various neutrinos of different energies that come from the Sun and distant Supernovae, that are produced through high-energy collisions in large accelerators and are revealed at a distance, all these facts needs a physical explanation. Might these evidences be linked to the fluctuations of the M-Q energy density originated by the high acceleration of colliding particles, as described at the end of par.12? In this case we might assign neutrino the nature of inertial wave propagating the local oscillations of the M-Q energy density produced by strong accelerations (collisions) of particles.

Some support to these conjectures comes from the unexpected electron-neutrino low flux from the Sun. The first experiment adopting a chlorine tank (Davis, 1964) sensible to low-energy neutrino found only $1 / 3$ of the expected flux. Successively the american-japanese experimental collaboration Kamiokande adopting water tank detectors, found also high-energy neutrinos opening an ample debate on the neutrino "oscillations" (Bahcall, 2004). Recently the Borexino experiment reported low-energy neutrinos coming from the "pep" thermonuclear reactions, which are probably the only way to produce low-energy neutrinos. The non existence of the neutrinos coming from natural beta decay may explain why the neutrinos from Sun continue to be less than expected. According to I. Anicin "the limits of neutrino parametres are narrowing, but the true nature of the neutrino remains uncertain" (Anicin, 2005).

\section{References}

Anicin, I. (2005). The neutrino: its past, present and future. arxiv:physics/0503172.

Bachall, J. (2004). Solving the Mystery of the Missing Neutrinos. Retrieved from http://www.nobelprize.org/nobel_prizes/physics//articles/bahcall/index.html

Brans, C. (2010).Varying Newton's constant: A personal history of scalar-tensor theories. Einstein online, 4, 1002.

Brans, C., \& Dicke, R., (1961). Mach's Principle and A Relativistic Theory of Gravitation. Physical Review, 124(3), 925. http://dx.doi.org/10.1103/PhysRev.124.925

Davis, R. jr. (1964). Solar Neutrinos. II Experimental. Phys. Rev. Lett. 12, 303-305. http://dx.doi.org/10.1103/PhysRevLett.12.303

Dirac, P. A. (1978). The large Numbers Hypothesis and the Einstein theory of gravitation. Proc. R. Soc. London, A.365, 19-30, Ed. 1979 (first Edition 1938).

Kuhn, T. S. (1962). The Structure of scientific revolutions. University of Chicago Press.

Kuhn, T. S. (2000). The road since Structure. Philosophical Essays 1970-1993. University of Chicago Press.

Loinger, A. (2007). Gravitational waves towards fundamental principles of General Relativity. arxiv; physics/0709.0490.

Michelini, M. (2008). The common physical Origin of the Gravitational, Strong and Weak force. Apeiron Journal, 15(4).

Michelini, M. (2010a). Major Gravitational Phenomena Explained by the Micro-quanta paradigm. Progress in Physics, 1. 
Michelini, M. (2011). The Gravitational power originating the Earthquakes and the Dansgaard-Oeschger catastrophic Events. Applied Physics Research, 3(1).

Michelini, M. (2010b). Physical Phenomena and Theoretical Problems explained by the Micro-quanta Paradigm, Applied Physics Research, 2(2).

Naeye, R. (2008). Testing fundamental Physics. NASA-FermiLab Gamma Ray Space Telescope, Aug. 2008

Perlmutter, S. et al. (1999). Measurements of $\Omega$ and $\Lambda$ from 42 high-redshift supernovae, Astrophysics J., 517 , 565-586. http://dx.doi.org/10.1086/307221

Rathbun, J. et al. (2003). Mapping of Io's thermal radiation by the Galileo photopolarimeter-radiometer instrument. Icarus, 169(1), 127-129. http://dx.doi.org/10.1016/j.icarus.2003.12.021

Riess, A. G. et al. (1998). Observational evidence from supernovae for an accelerating universe and a cosmological constant. Astronomical J., 116, 1009-1038. http://dx.doi.org/10.1086/300499

Riess, A. G. et al. (2004). Type Ia supernova discoveries at $\mathrm{z}>1$ from the Hubble Space Telescope : Evidence for past deceleration and constrains on dark energy evolution. Astrophysics J., 607, 665-687. http://dx.doi.org/10.1086/383612

Schoedel, R. et al. (2002). A star in a 15.2 years orbit around a supermassive black hole at the centre of Milky Way. Nature, 419, 694-696. http://dx.doi.org/10.1038/nature01121

Smolin, L. (2006). The Troubles with Physics. New York: Houghton Mifflin Company.

Stein, C. (1995). Global Earth Physics. Amer. Geoph. Union. 